Armed Forces Medical Services Saw it Coming Two Decades Back!

Dear Sir,

Tn a paper published recently [1] Choudhry et al from Harvard Medical School, systematically reviewed nearly 40 years of research on how medical knowledge and healthcare quality relate to years of clinical experience. The results should be considered an eye-opener. The performance of long-practicing physicians was worse than that of less-experienced physicians on all measures in $52 \%$ studies (32 studies). In additional $21 \%$ studies (13studies), long-practicing physicians performed worse on some measures, with no experience-related difference on other measures. Physicians younger than 40 years of age were more likely to correctly believe in the value of therapies that improve survival (for example, thrombolytic agents, aspirin and beta-blockers). They were also significantly less likely to believe in the value of therapies that have been disapproved (for example, prophylactic lidocaine for preventing ventricular tachycardia after myocardial infarction). It is mainly because medical advances occur frequently and the explicit knowledge that physicians possess may easily become out of date. Implications for Army Medical Corps can not be ignored as majority of our specialists are above 40 years of age. So next time you fall ill, do not search for the adviser, look for a graded specialist!

In early 1980s Armed Forces Medical Services decided to promote senior specialists to executive cadre and remove them from active patient care. It was a policy change praised by many though criticized by some. "You are removing specialists from active practice when they are most experienced!" "That is when a specialist really matters. You see, the best practicing doctors in civil are older ones!" This controversy was the subject of debate in many a coffee-room and bar-room discussion. Well! academic medicine has given its verdict in favour of the AFMS decision.

\section{Inappropriate Use of Hospital Beds.}

Dear Editor,

$\mathrm{T}$ his is with reference to the original article "Inappropriate use of hospital beds in a tertiary care service hospital", in MJAFI 2005 61:121-4 by Lt Col Abhijit Chakravarthy et al. The authors have carried out the study in a tertiary care hospital and found a rate of about $30 \%$ inappropriate hospitalization. Were the study to be carried out at a smaller hospital, the rate of inappropriate hospitalization would be a real eye opener. It would be higher due to various factors. Though the authors have not commented on it, the reasons for inappropriate occupancy of hospital beds could be

\section{Reply}

(a) It is appreciated that the issue of inappropriate utilization of hospital beds in service hospitals has received due attention from the esteemed readers of MJAFI.

(b) Inappropriate use of hospital beds may be the result of various reasons. In this study a validated AEP protocol was utilized and no over-riding factor was introduced to avoid observer bias. However, it will be interesting to repeat the study in other service hospitals.

(c) Staffing of service hospitals is dictated predominantly by authorized bed complement of the hospital. Inadequate staffing is because of ignoring OPD work-load as well as introducing new sub specialist centers without commensurate increase in
However, Choudhry's paper had failed to assess important measures like communication skills and intuitive analysis which are generally the strong point with older doctors. Interestingly this report showed that, on average, older doctors took better care of older and sicker patients. The accompanying editorial [2] comments that, practice alone does not make perfect but must be accompanied by continuing active effort to maintain competence. Even the organizations that provide continuing medical education must develop a bold vision of how to facitlitate professional development over a lifetime of practice. In the Armed Forces, study leave in the late 30 s or early 40 s does ensure professional enhancement for some specialists with several years of practice behind them.

Some argue that a professional capability report may be a good motivator for senior advisers to keep abreast of their profession. They forget that professional excellence remains a personal obsession with good specialists who see satisfying service to patients as an end in itself. Such specialists would keep in touch with recent advances despite service limitations. For others, there is always 'the executive cadre.'

\section{References}

1. Choudhry NK, Fletcher RH, Soumerai SB. Systematic review: The relationship between clinical experience and quality of health care. Ann Intern Med 2005 Feb 15; 142: 260-73.

2. Weinberger SE, Duffy D, Cassel CK. "Practice makes perfect"............. Or does it? Ann Intern Med 2005 Feb 15; 142: 302-3.

Col AC Anand, VSM*

*Professor and Head, Department of Medicine, Armed Forces Medical College, Pune-40.

the linking of hospital bed occupancy to the authorization of beds, staff strength and command structure.

A study to delink bed occupancy and staffing would probably decrease the incidence of inappropriate bed occupancy. Not only does inappropriate hospitalization tend to take the focus away from the needy patient, it also goes against the basic ethos of AMC to restore the fighting strength of the army

\section{Lt Col AP Gupta*}

*Classified Specialist (Anaesthesiology), Military Hospital, Golconda.

manpower.

(d) Bed Occupancy Rate (BOR) alone will not be sufficient for working out hospital utilization indices. Average length of stay and Turnover Interval need to be added for explaining the true utilization pattern of a service hospital.

(e) The author fully agrees that by artificially maintaining a higher BOR, the troops of the fighting arms are being denied their rightful place with the arms unit to which they belong to.

(f) The author is ready to provide necessary assistance to any reader who desires to conduct a similar study in any other hospital of the Armed Forces Medical Services.

Lt Col A Chakravarty*

"Officers Training School, AMC Center and School, Lucknow. 